\title{
Sistematización del programa de "Hábitos y estilos de vida saludables", desarrollado con trabajadores de la Fundación Hospital San Pedro
}

\section{Systematization of the program "Healthy habits and lifestyles", developed with workers of Fundación Hospital San Pedro}

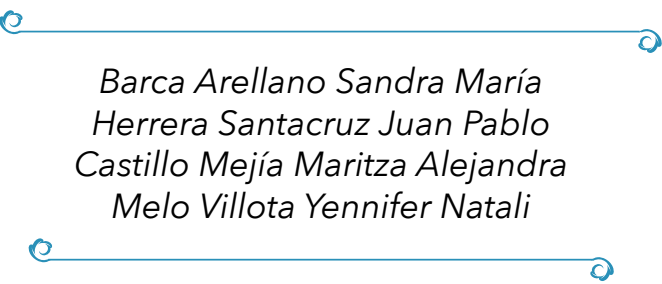

Recibido 08. 09. 2017 • Arbitrado 09. 10. 2017 •

Aprobado 15.11. 2017

Articulo presenta resultados de la investigación llevada a cabo para optar el título de psicólogo, Universidad de Nariño.

** Psicóloga -Universidad de Manizales, Magister en psicología- Universidad del Valle, especialista en Derechos Humanos y en Educación para el Aprendizaje Autónomo, Docente Universitaria del Departamento de Psicología de la Universidad de Nariño, asesora y coordinadora de proyectos y entidades públicas y privadas, docente universidades Públicas y Privadas. Pasto, Colombia, samabarc@gmail.com

*** Psicólogo -Pontificia Universidad Javeriana. Magister en Sistemas Integrados de Gestión de la Prevención de Riesgos Laborales- UNIR, España. Especialista en Derecho Laboral y Relaciones Industriales, Universidad Externado de Colombia. Docente departamento de Psicología Universidad de Nariño. Pasto, Colombia, juanpabloherrerasantacruz@gmail.com

**** Egresada del programa de Psicología de la Universidad de Nariño, ale16c@ hotmail.com

${ }^{* * * * *}$ Egresada del programa de Psicología de la Universidad de Nariño, jennifermelo31@gmail.com

\section{Resumen}

El artículo presenta la sistematización de las estrategias y los logros del programa de hábitos y estilos de vida saludables (HEVS), como parte del proyecto de práctica profesional del Programa de Psicología de la Universidad de Nariño con trabajadores de la Fundación Hospital San Pedro de la ciudad de Pasto, a través de una estrategia metodológica que pretende convertir la experiencia práctica en objeto de estudio e interpretación teórica. La información resultante de las acciones realizadas durante el periodo 2013-2016 se organizó en las categorías denominadas "estrategias utilizadas para la promoción de hábitos y estilos de vida saludables" y "logros del programa" dando cuenta de las fortalezas y oportunidades de mejora a lo largo del proceso.

Palabras clave: Salud en trabajadores, Hábitos saludables, estilo de vida, sistematización. 


\section{Abstract}

This article presents the systematization of the strategies and achievements of the healthy habits and lifestyles' program as a part of the professional practice project of the Psychology program of the University of Nariño with some workers of Fundación Hospital San Pedro of Pasto, through a methodological strategy that aims to transform the practical experience into an object of study and theoretical interpretation. The resulting information of the actions done between 2013 and 2016 was organized in the categories named "strategies used to the promotion of the healthy habits and lifestyles" and "achievements of the program" showing the strengths and opportunities of improvement throughout the process.

Keywords: health in workers, healthy habits, lifestyle, systematization.

\section{Introducción}

Los programas de promoción y prevención de la salud en el lugar de trabajo se plantean a menudo como una expresión del interés de la empresa por la salud y el bienestar de sus trabajadores, puesto que desean optimizarlos debido a la vinculación que tienen con la productividad (Messite y Warshaw, 1998).

Atendiendo a lo expuesto, la Fundación Hospital San Pedro en convenio con la Universidad de Nariño ejecutan el macroproyecto de práctica profesional de Psicología "Fortalecimiento de estilos de vida y trabajo saludables como co-constructores de la calidad de vida" que comprende componentes de intervención psicosocial a trabajadores, a través del desarrollo de programas que buscan fortalecer las relaciones interpersonales y la salud laboral (Universidad de Nariño y Fundación Hospital San Pedro, 2013-2016).

Para la presente investigación se retomó el programa que tenía como objetivo promover Hábitos y estilos de vida saludable (HEVS), los cuales se entendieron como patrones de conducta, creencias y conocimientos de las personas para mantener, restablecer o mejorar su salud (Sánchez y Luna, 2015) y se presentaron en ejes de actividad física, hábitos de consumo, comportamientos de seguridad, redes sociales de apoyo y hábitos de higiene (Becoña, Vázquez y Oblitas, 2004; Acuña y Cortés, 2012; García, García y Vaca, 2014).

Para dar cumplimiento al objetivo del programa se llevaron a cabo actividades basadas en estrategias psicoeducativas y lúdicas que facilitaron el reconocimiento de recursos y la potencialización de habilidades; de estas acciones se obtuvieron resultados que son de interés para la academia y la organización, 
por ende, para este ejercicio investigativo se plantea el objetivo de sistematizar las estrategias y logros del programa durante los años 2013 hasta el 2016. La importancia de sistematizar esta experiencia reside en reconstruir los conocimientos que han surgido a partir de la práctica (Berdegué, Ocampo y Escobar, 2004; Ahumada, 2014), de tal forma que se desarrolle la capacidad para tomar mejores decisiones en las acciones futuras o se pueda replicar en otros contextos (Berdegué et al., 2004; Villavicencio, 2009).

\section{Orientaciones metodológicas de la sistematización}

Esta investigación se basó en el paradigma cualitativo, ya que buscó reconstruir el proceso y su significado (Hernández, Fernández y Baptista, 2006; Ruiz, 2012), además, se llevó a cabo desde método de sistematización, orientado por lo planteado por Peresson (1996) quien señala que la sistematización convierte la propia experiencia de un programa o proyecto en objeto de estudio e interpretación teórica, a la vez que es objeto de transformación; con algunos ajustes propios relacionados con el reconocimiento del contexto desde la práctica profesional, de tal forma que los agentes involucrados en el desarrollo de la experiencia fueron docentes responsables de la práctica profesional en el área organizacional, estudiantes practicantes y trabajadores participantes del programa.

El proceso se dividió en dos etapas, la primera se refirió a la planificación de la sistematización y la segunda fue la recuperación, análisis e interpretación de la misma. La primera constó de la definición del objetivo y la identificación de las categorías de sistematización, las cuales corresponden a estrategias para la promoción de hábitos y estilos de vida saludables y logros del programa; para la segunda, se hizo uso de técnicas de análisis documental, grupo focal y entrevista semiestructurada.

\section{Resultados de la sistematización}

Los resultados de la sistematización se organizaron en las categorías definidas en la fase de planificación. A continuación, se despliega un esquema en el que se relacionan las categorías y los ejes emergentes, que surgen de los discursos de los participantes y los registros documentales del proceso (ver Figura 1). 


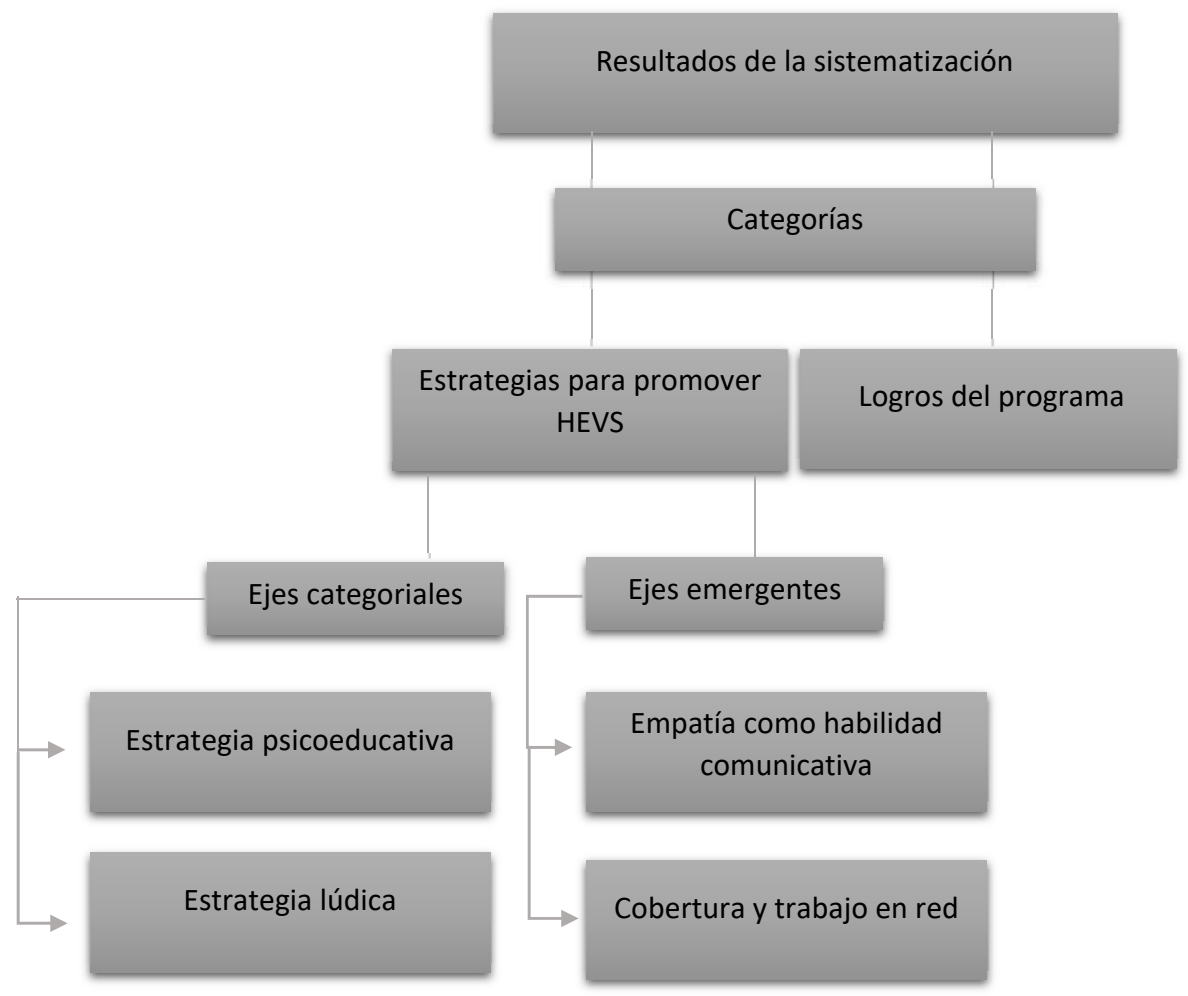

Figura 1. Resultados de la sistematización

Fuente: elaboración propia.

\section{Estrategias utilizadas para promocionar HEVS}

Las estrategias psicoeducativas y lúdicas desarrolladas a lo largo del programa se contemplaron como ejes categoriales de esta sistematización; sin embargo, se identificó que la empatía como estrategia comunicativa de los facilitadores y el trabajo en red fueron categorías emergentes que aportaron positivamente en el desarrollo del proceso. Estas estrategias se llevaron a cabo aproximadamente con 200 colaboradores de las áreas asistenciales y administrativas, ejecutándose 28 actividades con población variable en algunos casos, debido a la rotación constante del personal y la carga laboral.

\section{Estrategia psicoeducativa}

Cuando se hace uso de la psicoeducación las personas están en mejores condiciones para comprender y actuar positivamente sobre su salud, siempre y 
cuando se les brinde información clara y sencilla para su comprensión (Torres, 2008). En ese sentido, se observó que dicha estrategia se utilizó como un proceso de formación educativo para la salud que contribuyó al fortalecimiento de las capacidades individuales y el conocimiento en HEVS, apoyándose de técnicas como el taller, el juego de roles, la colcha de retazos, la relajación y jornadas informativas en conjunto con recursos didácticos como tarjetas, juegos, folletos o carteleras institucionales. Para esto, se llevó información hasta los puestos de trabajo en las áreas asistenciales y administrativas del hospital; los trabajadores y los facilitadores identificaron que realizar este tipo de acciones minimiza las implicaciones resultantes de retirar a las personas de sus ocupaciones en el hospital y resultan de interés para el colaborador, refiriendo,

Se ha evidenciado que los trabajadores participan más de las actividades psicoeducativas puesto que son cortas, concretas, llamativas y no interfieren con sus labores (F. 1). ${ }^{1}$

Me parece que son buenas las veces en que ustedes van con folletos o tarjetas hasta nuestro sitio de trabajo a darnos información, porque uno no puede salir a veces a las actividades grupales ya que hay mucho que hacer (T. 1). ${ }^{2}$

Pienso que manejar el tema de psicoeducación es importante porque es re-aprender cosas, porque empieza uno a borrar paradigmas que ya se había establecido y la idea es poder cambiarlos (T. 2).

Esto ocurre porque llevar a cabo esta estrategia no implica retirar a la persona de su oficina o área y no requiere largos lapsos de tiempo en sus actividades, tal como lo menciona Torres (2008), resulta económica en tiempo, espacio y recursos; de igual forma, se reconoce que la psicoeducación amplía la información que se tiene acerca de un tema, rompiendo así con lo que tradicionalmente ya se cree.

\section{Estrategia lúdica}

La lúdica se considera como una estrategia educativa que ayuda a desarrollar aptitudes de pensamiento lógico, táctico y creativo y fija metas de desarrollo

1 Información obtenida de facilitadores que hicieron parte del proceso de práctica durante los años 2013 a 2016 en la Fundación Hospital San Pedro, mediante entrevistas semi-estructuradas, grupo focal y reuniones de asesoría.

2 Información obtenida de trabajadores de la Fundación Hospital San pedro participantes del programa HEVS, durante los años 2013 a 2016, mediante entrevistas semi-estructuradas y registros documentales del proceso. 
relacionadas con la salud, la autonomía, el autocuidado y las buenas relaciones interpersonales (Tobón, Gaviria y Ramírez, 2012; Posada, 2014).

Se evidenció que esta estrategia incluyó el desarrollo de juegos típicos como el trompo, cubos, yoyos, rompecabezas, sapo, ping pong aéreo, yenga, juegos de mesa, concursos de fotografía, talleres de pintura y juegos modernos como el Nintendo Wii, con los cuales se promocionó la adopción de hábitos y estilos de vida saludables; lo anterior fue valorado positivamente por gran parte de los trabajadores, pues consideraban que era una forma alternativa y novedosa de generar aprendizajes en interacción con sus compañeros, a la vez, que recordaban actividades lúdicas de su infancia.

Cuando nos citan a una capacitación de dos horas, nos cansamos, se pierde la concentración y eso no sucede cuando se hacen cosas lúdicas, además que esas actividades casi no se ven en el trabajo y más aún en un hospital (T. 3).

La Lúdica nos da la posibilidad de compartir con nuestros compañeros y traer al presente lo que jugábamos cuando éramos niños (T. 4).

En ese sentido, se estima que la lúdica es un proceso inherente al desarrollo humano ligado a las prácticas cotidianas y pedagógicas que llevan a la comprensión y al aprendizaje (Posada, 2014), además, a través del ocio y la recreación, se disminuye la fatiga ocasionada por la rutina de actividades laborales y se promueve la apertura de interrelaciones que pueden generar cambios positivos (Tobón et al., 2012), que para este caso sería la adopción de acciones saludables. No obstante, es importante considerar que para algunas personas las actividades que implican juegos o recreación no resultan de interés y es necesario utilizar otro tipo de estrategias para realizar las acciones de promoción, como podrían ser la psicoeducación o la capacitación.

A mí no me gusta participar en juegos, yo prefiero las actividades que son informativas, de lectura o trabajo mental (T. 5).

\section{Empatía como estrategia comunicativa de los facilitadores.}

La empatía fue una estrategia emergente que se identificó a partir del análisis de la información, teniendo en cuenta que favoreció la comunicación entre facilitadores y trabajadores a partir de la creación de lazos emocionales que afectaron positivamente en la ejecución del programa, 
... fue un apoyo porque le daban a uno confianza, entonces era un logro muy grande la confianza y la relación interpersonal de ustedes con nosotros (T. 6).

Conviene subrayar que la empatía funciona como una habilidad comunicativa en la que debe existir escucha activa por parte del receptor y habilidades del emisor para hacerse comprender, generando libertad de expresión, claridad, continuidad, respeto y consideración (Muñoz y Chaves, 2013); igualmente, se caracteriza por la presencia de sentimientos de cercanía, facilitando el desarrollo óptimo de salud mental que se manifiesta en relaciones sociales satisfactorias (Martínez, 2006).

\section{Estrategia de trabajo en red.}

Forni, Castronuovo y Nardone (2007) afirman que el trabajo en red se desarrolla comúnmente entre organizaciones, no obstante es posible utilizar dicha dinámica al interior de una empresa, puesto que busca la articulación de esfuerzos solidarios entre diferentes áreas o personas, ampliar la comprensión de un tema o de una acción, generar acciones efectivas, ampliar la cobertura, evitar el desperdicio de recursos, facilitar procesos de aprendizaje y generar una capacidad instalada, para dar continuidad a los acciones y mejorarlas. En la sistematización se evidenció que para llevar a cabo el programa se realizaron alianzas con el Sistema de Gestión de Seguridad y Salud en el Trabajo y los coordinadores de cada área, lo que facilitó la logística, recursos, permisos, apoyo y asesoría profesional, esta dinámica se relaciona con trabajar en red. Aunque se infiere que esta estrategia puede ayudar a ampliar aún más la cobertura del programa dado que algunos trabajadores perciben que la participación es baja,

...la participación de compañeros en las actividades es baja, es entendible que no es fácil sacar al personal asistencial para que participe siempre ( $\mathrm{T}$. 7).

... hay que llegar a más personas, por ejemplo en mi área, entre cambio de sala hay unos minutos que se pueden aprovechar, hacer grupos pequeños en las diferentes jornadas (T.8).

Lo anterior, lleva a comprender que el trabajo en red que se realizó se debe fortalecer para cubrir las necesidades del personal sobre todo en el área asistencial, considerando que en la mayoría de casos sus labores requieren a las personas en la totalidad de su tiempo, por ello es importante aprovechar o 
crear nuevos espacios extralaborales de forma óptima para el desarrollo del programa.

\section{Logros del programa}

Los logros se identificaron teniendo en cuenta los ejes para abordar HEVS como son participación en redes de apoyo, comportamientos de seguridad, hábitos de higiene, nutrición, ejercicio físico y consumo de sustancias psicoactivas. En el desarrollo de los ejes los trabajadores generaron compromisos a corto, mediano y largo plazo, orientados a poner en práctica dichos hábitos y disminuir la aparición de factores de riesgo.

En relación a la participación social en redes de apoyo se identificó que se encuentran la red de trabajo y la red familiar; de la primera red se mostró que los participantes verbalizaron la importancia de establecer buenas relaciones con sus compañeros y practicar valores como el respeto, la solidaridad y la confianza; Asimismo, refirieron la necesidad de generar espacios propicios para fortalecer las relaciones interpersonales en el trabajo, a través de actividades institucionales en las que se fomente la interacción e integración de trabajadores.

Aprendí que es importante la unión entre los trabajadores, tratarnos con respeto y conocerse a nivel personal, para mejorar la relación entre jefe y el trabajador (T. 9)

Sería interesante que en su programa hicieran actividades deportivas para integrar a las personas, para conocerlas mejor,... (T. 10).

Con respecto a las expresiones de los participantes, García et al. (2014), señalan que las personas pasan gran parte de su tiempo laborando, por lo que fortalecer la red de trabajo a partir de la interacción entre los trabajadores es una de las formas para aportar a su bienestar. En relación a la segunda red, a través de la promoción del buen trato entre madre e hijo, las trabajadoras expresaron emociones y sentimientos de amor, nostalgia, gratitud y en ocasiones culpa por no compartir tiempo suficiente con ellos.

Soy la mejor mamá del mundo porque cuido a mis hijos, los quiero, les enseño valores, pero me gustaría estar más tiempo con ellos, jugar y hacer tareas juntos (T.11). 
Lo anterior sugiere que las jornadas laborales pueden interferir con la relación familiar, por lo cual es necesario continuar con acciones que generen espacios propicios de calidad en familia, tal como lo afirma García et al. (2014), la red familiar es una de las fuerzas promotoras de la salud que brindan apoyo instrumental y cumplen funciones en el desarrollo biológico, psíquico y social de la persona, por tanto, fortalecer estos vínculos, afectaría positivamente en el desempeño de los trabajadores del Hospital.

A su vez, se denota que los trabajadores reconocieron que desarrollar comportamientos seguros en los lugares de trabajo disminuye la posibilidad de presentar una lesión o patología, particularmente los participantes de las áreas asistenciales refirieron que la carga laboral y mental suelen ser un factor desencadenante de estrés, en relación a esto consideran que realizar pausas laborales activas durante las jornadas aporta a su bienestar.

Las pausas para mí son descansos, pues yo he estado muy estresado en ciertos momentos, el trabajo, la gente y llegaban ustedes como a despejar un poco la mente y eso me hacía sentir bien (T. 12).

Es por ello que realizar breves descansos durante la jornada laboral, hace que las personas recuperen energías y mejoren su desempeño, a través de diferentes técnicas y ejercicios que ayuden a reducir la fatiga y tensión laboral, enfermedades profesionales y prevenir el estrés (Bonilla, 2012; Cañas, 2016).

Otro aspecto abordado fue, hábitos de higiene del sueño, el cual evidenció que los trabajadores realizan prácticas inadecuadas que alteran sus espacios de descanso, como por ejemplo ver programas de televisión violentos antes de ir a dormir o comer en sus habitaciones, de igual forma, por sus horarios de trabajo tienen dificultades para conciliar el sueño al menos ocho horas continuas durante la noche o el día; un hábito saludable contempla un tiempo y espacio de descanso adecuados, como lo menciona Oblitas (2008).

Con los turnos de trabajo uno no puede descansar, porque a veces tiene que trabajar en la noche y llegar a dormir a la casa en el día (T. 13).

En el día uno deja de dormir y se pone a hacer otras cosas y cuando se llega de nuevo al trabajo uno se siente cansado, como adormecido y entonces tiene que tomar algún energizante (sic) o un café bien cargado (T. 14). 
En conformidad a lo anterior, Alferez y Matta (2013) indican que gran parte de los trabajadores que se desempeñan en turnos de trabajo se ven expuestos a condiciones laborales desfavorables para su bienestar, afectando su salud física y mental. Como se identifica en el discurso de los participantes y de acuerdo con los autores mencionados, este tipo de trabajadores pueden llegar a presentar insomnio y somnolencia, teniendo mayor dificultad para realizar sus tareas, con disminución del rendimiento laboral y aumento en el riesgo de accidentes. Por esto, es importante que se generen estrategias de intervención que minimicen los efectos negativos que puedan producirse en la vida diaria de los trabajadores que asumen estas circunstancias, y entre dichas estrategias se encuentran la realización de investigaciones que recojan las fortalezas y debilidades con las que cuentan las instituciones a la hora de abordar el tema, la implementación de periodos de siesta y establecimiento de tiempos de descanso, la planeación adecuada de la forma de rotación de los turnos y la intervención psicológica (Feo, 2008).

$\mathrm{Al}$ presentarse dichas alteraciones del sueño y otros factores de riesgo psicosocial intralaboral y extralaboral con consecuencias como, estrés, dificultades de relaciones y bajo desempeño en el trabajo o inadecuadas relaciones en el hogar, los trabajadores adoptan consumo de sustancias nocivas para su salud, entre los que se encuentran bebidas energizantes, analgésicos auto-medicados, alcohol, cigarrillo y otras sustancias psicoactivas (Lara y Vargas, 2010), en efecto, el programa muestra que se desarrollaron actividades de promoción frente al consumo saludable, entre ellas, elaboración y degustación de cócteles sin alcohol, jornadas informativas acerca de los efectos nocivos de las sustancias psicoactivas. En el discurso de los colaboradores se reflejó el aporte positivo a generar mayor sensibilización y conciencia frente a la salud, refiriendo,

Me enseña a tener como más consciencia de mí ser, del cuidado de mi salud y de mi cuerpo (T. 15).

Así mismo, se encontró promoción de beneficios al realizar actividad física, deporte y recreación, pues como lo refiere Oblitas (2008) practicar ejercicio físico de forma regular tiende a prevenir la aparición de alteraciones físicas y psicológicas, esto contribuye a la estabilidad emocional de las personas, fruto de la reducción de la ansiedad y la tensión, de igual forma, González, Huéscar y Moreno (2013) señalan que la práctica de actividad deportiva repercute en la percepción sobre la satisfacción con la vida, mejorando la percepción de calidad de vida, procura un envejecimiento saludable, incrementa el sentimiento de bienestar y reduce el estrés; es así que las actividades ejecutadas 
con relación a estos aspectos fueron valoradas de forma positiva por los participantes, quienes identificaron beneficios y percibieron satisfacción a través de la realización de actividad física.

Hacer deporte me relaja, me libera de tensiones y me hace sentir una energía nueva (T16).

Del mismo modo, se encontraron acciones de fomento por el consumo de alimentos y bebidas saludables, los cuales, de acuerdo con García et al. (2014) satisfacen las necesidades nutritivas que mejoran el desempeño de las personas; como la promoción de ingesta de frutas y agua, la disminución del consumo de grasas, azucares y bebidas energizantes; que se desarrollaron a través de jornadas psicoeducativas acompañadas de pequeñas muestras de alimentos saludables. Frente a esto, se evidenció que las personas intervenidas asumen la importancia de desarrollar hábitos y estilos saludables relacionados con la alimentación.

No deberíamos tener jugos artificiales ni energizantes ni nada de esos productos en la cafetería y en las máquinas, o sea tendría que ser una cosa coordinada y coherente (T. 17).

Con respecto a lo anterior, Santacoloma y Quiroga (2009) refieren que las variables ambientales pueden jugar un papel importante a la hora de establecer la conducta alimentaria, por lo cual la disposición de alimentos poco saludables al interior del hospital es un factor de riesgo que se encontró en la ejecución del programa.

\section{Conclusiones y recomendaciones}

La sistematización permitió comprender cómo las estrategias utilizadas fueron oportunas en cuanto a espacio y tiempo, evidenciando que la psicoeducación permite acceder a las personas sin alterar su ritmo de trabajo, y la lúdica se percibe como una estrategia motivacional y de satisfacción.

A partir de la sistematización se generaron dos categorías emergentes, las cuales se denominaron empatía como habilidad comunicativa y trabajo en red. Frente a ello, la primera facilitó la comunicación entre facilitadores y trabajadores, mientras que la segunda puede fortalecerse al ampliar la cobertura para optimizar las acciones a través del trabajo en red, es decir, continuar la articulación con el Sistema de Gestión de Seguridad y Salud en el trabajo, con 
los coordinadores de cada área e incluir en la red a la coordinación de Talento Humano, al comité de Bienestar Laboral y agentes externos como la familia de los trabajadores; en la misma medida, es necesario que se fortalezca la sensibilización en cuanto a la participación y la importancia de hacer parte de los programas de promoción de la salud.

Igualmente, se observó que se cumplió el objetivo del programa, al lograr que los participantes hagan conciencia de los beneficios de practicar HEVS y consideren fortalecer la realización de actividad física, la participación en redes sociales de apoyo y la alimentación y consumo saludable; de igual forma a partir de los resultados se identificó que es necesario generar acciones de mejoramiento en cuanto a variables que afectan la higiene del sueño como son los turnos de trabajo.

Finalmente, con base en los resultados de esta sistematización y realizando los ajustes pertinentes en el plan de acción del programa, a futuro se podría crear una política institucional acerca de hábitos y estilos de vida saludables, para reconocer, evaluar y controlar los riesgos de la salud de los trabajadores.

\section{Agradecimientos}

A la Universidad de Nariño y a la Fundación Hospital San Pedro ubicadas en la ciudad de Pasto - Nariño, por su compromiso académico y social; a docentes, estudiantes y trabajadores que hicieron parte del proyecto de práctica profesional durante los años 2013 a 2016.

\section{Referencias}

Acuña, Y., Cortés, B. (2012). Promoción de estilo de vida saludable área de salud de Esparza. (Tesis de Maestría). Instituto Centroamericano de Administración Pública, San José, Costa Rica. Recuperado de http://biblioteca.icap.ac.cr/BLIVI/ TESIS/2012/acuna_castro_yessika_sa_2012.pdf

Ahumada, E. (2014). Sistematización de experiencias de la práctica profesional de los estudiantes de Psicología de la UNAD, en la ONG Grupos Humanos. Universidad Nacional Abierta y a Distancia. Zipaquirá, Colombia. Recuperado dehttp://stadium. unad.edu.co/preview/UNAD.php?url=/bitstream/10596/2535/1/20985996.pdf

Alferez, L., Matta, H- (2013). Trastornos del sueño y sus efectos en trabajadores de la salud del área asistencial: revisión de investigaciones publicadas en el periodo 1990-2013. (Tesis de posgrado). Universidad de Antioquia, Medellín, Colombia. Recuperado de_http://www.udea.edu.co/wps/wcm/connect/udea/8df1d1be- 
8bab-4b89-b881-a8f495fda828/trastornos + del+sue\%C3\%B1o+y+su+efectos. pdf?MOD=AJPERES

Becoña, E., Vázquez, F., y Oblitas, L. (2004). Promoción de los estilos de vida saludables. Alapsa, 5,1-40. Recuperado de http://salud.edomex.gob.mx/html/ MEDICA/Psicologia\%20Estilos\%20de\%20vida\%20saludables.pdf

Berdegué, J., Ocampo, A. y Escobar, G. (2004). Sistematización de experiencias locales de desarrollo agrícola y rural. Guías de Terreno. Recuperado de http://200.110.137.61/wp/wpcontent/uploads/2010/02/guiadeterrenodesiste matizacionfidamericapreval.pdf

Bonilla, F. (2012). Propuesta de un programa de pausas activas para colaboradores que realizan funciones de oficina en la empresa de servicios públicos Gases de Occidente S.A E.S.P de la ciudad de Cali (Tesis de pregrado). Universidad del Valle, Cali, Colombia. Recuperado de http://bibliotecadigital.univalle.edu.co/ handle/10893/4370

Cañas, R. (2016). Implementar un programa de pausa activa a los empleados del club campestre El Rancho para mejorar la calidad de vida y prevenir las enfermedades profesionales. Actividad física y deporte, 2 (1) 5-21. Recuperado de_https://www. udca.edu.co/wp-content/uploads/revista-deportes/revista-digital-actividadfisica-deporte-vol2-no1.pdf

Feo, J. (2008). Influencia del trabajo por turnos en la salud y la vida cotidiana. (Tesis de posgrado). Pontificia Universidad Javeriana, Bogotá, Colombia. Recuperado de http://www.javeriana.edu.co/biblos/tesis/enfermeria/tesis38.pdf

Forni, P., Castronuovo, L., Nardone, M. (2007). Las organizaciones en red y la generación de capital social. Implicancias para el desarrollo comunitario. Miriada, 8, 79-106. Recuperado de_https://dialnet.unirioja.es/descarga/articulo/5024489. pdf

García, J., García, C., Vaca, M. (2014). Variables involucradas en la práctica individual y colectiva de hábitos saludables. Revista de salud pública, 16 (5), 719732. Recuperado de http://www.scielosp.org/pdf/rsap/v16n5/v16n5a07.pdf

González, G., Huescar, E., Moreno, J. (2013). Satisfacción con la vida y ejercicio físico. Motricidad. European Journal of Human Movement, 30, 131-151. Recuperado de http://www.redalyc.org/pdf/2742/274228060010.pdf

Hernández, R. Fernández, C., Baptista, P. (2006). Metodología de la investigación (4ta. ed.). México D.F.: Editorial McGraw-Hill.

Lara, C., Vargas, G. (2010). Consumo de sustancias psicoactivas en profesionales de la salud (médicos y enfermeros) de dos IPS de primer nivel de atención en 
consulta externa de Bogotá. (Tesis de posgrado). Universidad del Rosario, Bogotá, Colombia. Recuperado de http://repository.urosario.edu.co/bitstream/ handle/10336/2335/527782482011.pdf? sequence $=1$

Martínez, M. (2006). El estudio científico de las fortalezas trascendentales desde la Psicología Positiva. Clínica y Salud, 17(3), 245-258. Recuperado de_http://scielo. isciii.es/scielo.php?script $=$ sci_arttext\&pid $=$ S1130-52742006000300003

Messite, J., Warshaw, L. (1998). Protección y promoción de la salud. En Mager, J. (Ed.), Enciclopedia de seguridad y salud en el trabajo. 152-245. Madrid, España: Ministerio de Trabajo y Asuntos Sociales, Subdirección General de Publicaciones. Recuperado de http://www.insht.es/InshtWeb/Contenidos/Documentacion/ TextosOnline/EnciclopediaOIT/tomo1/15.pdf

Muñoz, A., Chaves, L. (2013). La empatía: ¿un concepto unívoco? Katharsis, 16, 123143. doi: http://dx.doi.org/10.25057/25005731.467

Oblitas, L. (2008). Psicología de la salud: una ciencia de la felicidad y el bienestar. $A v$. Psicol, 16(1), 9-38. Recuperado de http://www.unife.edu.pe/pub/revpsicologia/ psicologiasalud.pdf

Peresson, M. (1996). Metodología de un proceso de Sistematización. Pasos Fundamentales del proceso de sistematización del proyecto y experiencia de teología popular, 1985-1995. Aportes, 44.

Posada, R. (2014). La lúdica como estrategia didáctica. (Tesis de Maestría). Universidad Nacional de Colombia, Bogotá, Colombia. Recuperado de http://www.bdigital. unal.edu.co/41019/1/04868267.2014.pdf

Ruiz, J. (2012). Metodología de la investigación cualitativa (5ª . Ed.). España: Deusto.

Sánchez, M., Luna, E. (2015). Hábitos de vida saludable en la población universitaria. Nutrición Hospitalaria, 31(5), 1910-1919. doi: 10.3305/nh.2015.31.5.8608

Santacoloma, A., Quiroga, L. (2009). Perspectivas de estudio de la conducta alimentaria. Revista Iberoamericana de Psicología: ciencia y tecnología, 2 (2), 7-15. Recuperado de http://revistas.iberoamericana.edu.co/index.php/ripsicologia/ article/view/180/314

Tobón, F., Gaviria, N., Ramírez, J. (2012). La lúdica como método psicopedagógico: una experiencia para prevenir farmacodependencia en jóvenes. Avances en Psicología Latinoamericana, 30(1), 81-92. Recuperado de http://www.scielo.org. co/pdf/apl/v30n1/v30n1a07.pdf

Torres, N. (2008). Psicoeducación. En C, Gómez (Ed.), Psiquiatría clínica. Diagnóstico y tratamiento en niños, adolescentes y adultos (pp. 683-685). Bogotá, Colombia: 
Editorial Médica Internacional Ltda. (pp.681-686). Bogotá, Cundinamarca: Editorial Médica Ltda.

Universidad de Nariño y Fundación Hospital San Pedro (2013-2016). Fortalecimiento de estilos de vida y trabajo saludables como co-constructores de la calidad de vida. Proyecto de práctica profesional de Psicología, Pasto, Colombia.

Villavicencio, R. (2009). Manual autoinstructivo: aprendiendo a sistematizar las experiencias como fuentes de conocimiento. Recuperado de http://www. bibliotecavirtual.info/wp-content/uploads/2013/08/Aprendiendo-asistematizar.pdf 